\title{
Sterol upregulation of human CETP expression in vitro and in transgenic mice by an LXR element
}

\author{
Yi Luo and Alan R. Tall \\ Division of Molecular Medicine, Department of Medicine, Columbia University, New York, New York 10032, USA
}

Address correspondence to: Alan R. Tall, Division of Molecular Medicine, Department of Medicine, Columbia University, New York, New York 10032, USA. Phone: (212) 305-4899; Fax: (212) 305-5052; E-mail: art1@columbia.edu.

Received for publication September 29, 1999, and accepted in revised form December 30, 1999.

\begin{abstract}
The cholesterol ester transfer protein (CETP) facilitates the transfer of HDL cholesterol esters from plasma to the liver. Transgenic mice expressing human CETP, controlled by its natural flanking region, increase expression of this gene in response to hypercholesterolemia. We established a CETP promoter-luciferase reporter assay in differentiated 3T3-L1 adipocytes to map the sterol upregulatory element. Promoter mutagenesis suggested that a direct repeat of a nuclear receptor binding sequence separated by 4 nucleotides (DR4 element, -384 to -399) was responsible for this activity. Using mice carrying normal or mutated promoter sequences, we confirmed the importance of this element for gene induction by dietary sterol. A gel retardation complex containing LXR/RXR was identified using the CETP DR4 element and adipocyte nuclear extracts. Both LXR $\alpha / R X R \alpha$ and $\mathrm{LXR} \beta / \mathrm{RXR} \alpha$ transactivated the CETP promoter via its DR4 element in a sterol-responsive fashion. Thus, the positive sterol response of the CETP gene is mediated by a nuclear receptor binding site that is activated by LXRs. That Cyp7a, the rate-limiting enzyme for conversion of cholesterol into bile acids in the liver, is also regulated by LXR $\alpha$ suggests that this class of nuclear receptor coordinates the regulation of HDL cholesterol ester catabolism and bile acid synthesis in the liver.
\end{abstract}

J. Clin. Invest. 105:513-520 (2000).

\section{Introduction}

The cholesterol ester transfer protein (CETP) is a 74$\mathrm{kDa}$ glycoprotein that mediates the transfer of cholesterol esters (CEs) from HDL to triglyceride-rich lipoproteins (1), which are subsequently cleared in the liver. The importance of CETP in HDL metabolism has been elucidated by human genetic deficiency of CETP $(2,3)$ and by CETP transgenic mice $(4,5)$. In human genetic CETP deficiency, HDL levels are increased but there appears to be an excess of coronary heart disease (6). Conversely, in hypertriglyceridemic CETP transgenic mice, CETP expression decreases atherosclerosis while lowering overall HDL levels (7). These findings suggest that reverse cholesterol transport facilitated by $\operatorname{CETP}(8,9)$ has an antiatherogenic role.

In humans and animals, plasma CETP and tissue mRNA levels are increased in response to high-fat, highcholesterol diets (10). CETP is synthesized in liver and small intestine as well as a number of peripheral tissues, notably adipose $(1,11,12)$. Mice do not have plasma CETP activity. However, human natural flanking region (NFR) CETP transgenic mice show a humanlike pattern of tissue expression and an increase in plasma CETP activity and mass in response to a high-fat, high-cholesterol diet (13). Moreover, in response to endogenous hypercholesterolemia in LDL receptor and apoE knockout mice, the plasma CETP levels and hepatic CETP mRNA are dramatically induced (4- to 10-fold). These increases are due to elevated CETP gene transcription in liver and peripheral tissues (5). There is a strong corre- lation between plasma cholesterol and plasma CETP in both CETP transgenic mice and humans $(13,14)$. These studies also indicate that the CETP gene expression is driven by a mechanism that senses high plasma cholesterol levels independent of apoE and LDL receptors (5).

A number of other genes have been shown to be upregulated by increased cellular cholesterol stores $(15,16)$. However, relatively little is known concerning the molecular mechanisms governing sterol upregulation of these genes. Several orphan nuclear receptors, including LXR $\alpha$ (NR1H3), LXR $\beta$ (NR1H2), and SF-1 (NR5A1), are activated by sterols in cell culture (17-20). LXRs were initially identified as orphan nuclear receptors (Liver X receptor) by screening libraries for homologues of nuclear hormone receptors (21-23) and were subsequently shown to be activated by hydroxy sterols at physiological concentrations $(17,18)$. An LXR $\alpha$ binding site was identified in the promoter of the Cyp7a gene, encoding cholesterol $7 \alpha$-hydroxylase, the first rate-limiting enzyme in the pathway converting cholesterol to bile acids. LXR $\alpha$ was found to transactivate the Cyp7a promoter (19). Furthermore, disruption of LXR $\alpha$ in mice abolished induction of Cyp7a expression by dietary cholesterol (24). However, Cyp7a is the only gene that has been identified as a direct target of LXR $\alpha$. Although LXR $\beta$ is likely to play a distinctive role in gene regulation (24), its targets and function are poorly understood.

To localize the promoter elements responsible for increased CETP expression in response to hypercholesterolemia, CETP transgenic mice containing different 
$\mathbf{a}$

a

a
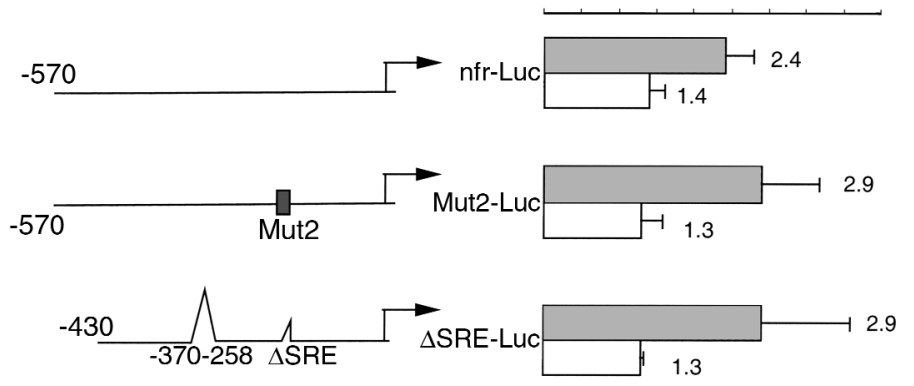

b

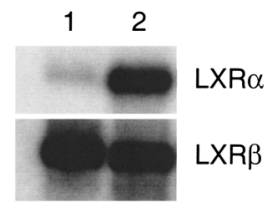

Figure 1

(a) Induction of the CETP promoter in adipocytes (shaded bar) but not preadipocytes (open bar). 3T3-L1 preadipocytes and differentiated adipocytes were transfected with reporter constructs in which the luciferase reporter gene is controlled by the proximal CETP promoter (from 1 to -570 ) with or without mutation in the SRE-like element (Mut2). The transfected cells were cultured in LPDS or LPDS $+4 \mu \mathrm{g} / \mathrm{mL} 22(\mathrm{R})$ hydroxycholesterol for 24 hours. Luciferase activities were measured using the Promega Dual-Luciferase assay system, which uses Renilla luciferase as a control for transfection efficiency. The fold induction by sterol was calculated by dividing the luciferase activity in the presence of sterol by the activity in the presence of vehicle only. Means \pm SD are shown. (b) RNA expression of LXR $\alpha$ and LXR $\beta$ in 3T3-L1 preadipocytes (lane 1) and differentiated adipocytes (lane 2). Northern blots were performed with equal amount of Poly $A^{+}$RNA isolated from 3T3-L1 preadipocytes and day 4 differentiated adipocytes using LXR $\alpha$ and LXR $\beta$ cDNA probe $(21,37)$.

lengths of natural flanking sequences were prepared (25). The transgenesis approach was used because of the difficulty in obtaining significant sterol responses of CETP promoter controlled reporter genes in several cultured cell lines $(25,26)$. The in vivo analysis localized the sterol upregulatory element (SURE) to a region between -138 and -570 in the human CETP promoter. We have recently reinvestigated the regulation of CETP promoter-reporter constructs in cell culture and discovered that positive sterol response could be obtained in differentiated adipocytes. This enabled us to fine map the SURE and subsequently to confirm the in vivo significance of this element by mutation in NFR-CETP Tg mice.

\section{Methods}

Cell culture and transfection. The 3T3-L1 cell line (American Type Culture Collection, Rockville, Maryland, USA) was maintained in DMEM with $10 \%$ FBS at $37^{\circ} \mathrm{C}$. Confluent cells were induced to differentiate as described elsewhere (27). Day 4-6 differentiated cells cultured in 6-well plates were transfected using
MBS calcium phosphate precipitation (Stratagene, La Jolla, California, USA). A total of $2.5 \mu \mathrm{g}$ of reporter DNA and $1 \mu \mathrm{g}$ of TK-RL (Renilla) (Promega Corp., Madison, Wisconsin, USA) were cotransfected into cells. CV-1 cells cultured in 24-well plates were transfected using Lipofectamine transfection reagent (Life Technologies, Grand Island, New York, USA). A total of $0.3 \mu \mathrm{g}$ of reporter DNA, $25 \mathrm{ng}$ of pCMV-RL, $50 \mathrm{ng}$ of receptors (CMX-hRXR $\alpha$, CMX-hLXR $\alpha$, and CMVLXR $\beta$ ), or 50 ng of PCMV vector was used. The transfected cells were cultured in lipoprotein-dificient serum (LPDS) medium in the presence of $4 \mu \mathrm{g} / \mathrm{mL}$ 22(R)-hydroxycholesterol (Sigma Chemical Co., St. Louis, Missouri, USA), $1 \mu \mathrm{M}$ 9-cis retinoic acid (Biomol Research Laboratories, Plymouth Meeting, Pennsylvania, USA), or vehicle alone for 24 hours. The luciferase activities were measured using Promega Dual Luciferase assay system. Reporter constructs used to analyze the promoter region were constructed by subcloning PCR fragments containing deleted or mutated CETP promoter regions into pGL3-luc

\section{Figure 2}

Deletional analysis of the CETP promoter in adipocytes. Reporter constructs containing various truncated proximal CETP promoter were transfected into 3T3-L1 adipocytes. The fold induction (means $\pm \mathrm{SD}$ ) by $22(\mathrm{R})$-hydroxycholesterol is shown. ${ }^{*} P<0.005$ versus -60 Luc; ${ }^{*} P<0.0005$ versus -60 -Luc).

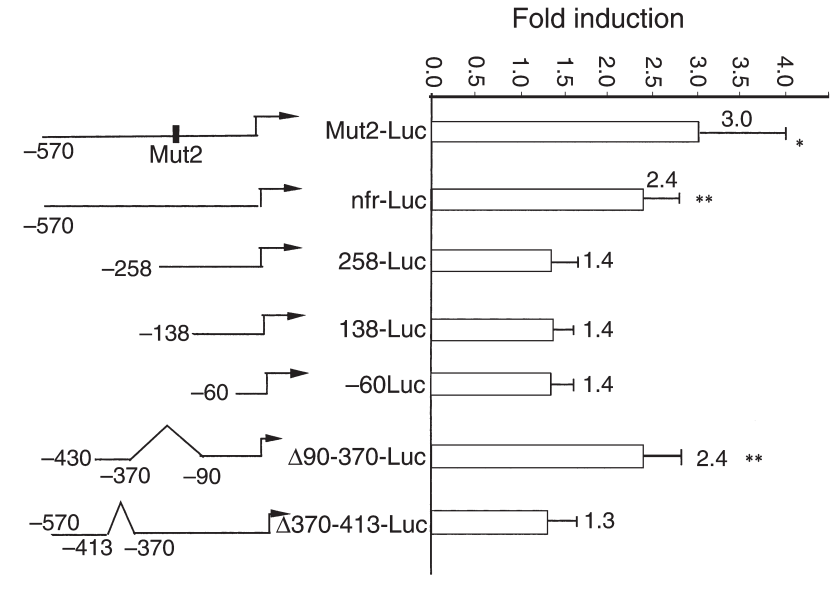


basic vector (Promega). Oligonucleotides containing 3 copies of the CETP DR 4 elements or 3 copies of the $5^{\prime}$ half of the CETP DR4 elements were annealed and subcloned into TK-Luc vector (21) to create $3 \times c$ DDR4-Luc and $3 \times 1 / 2$ cpDR4-TK-Luc.

Transgenic mice dietary studies and plasma CETP activity assays. Mut3-CETP, M1-CETP, and M3-CETP plasmids, created using the megaprimer PCR method (28), were digested with NotI/SalI, and the 10-kb transgene fragments were isolated by agarose gel electrophoresis and purified by $\mathrm{CsCl}$ gradient fractionation. Transgenic mice were generated and screened as described elsewhere (28). Transgenic founders or F1 mice were maintained on a chow diet (Purina Chow 5001; Purina Mills Inc., St. Louis, Missouri, USA). Five MUT3-CETP founders (1 female and 4 male), 4 MUT3-CETP F1 (male), $2 \mathrm{M} 1{ }^{*}$-CETP founders (female), and $2 \mathrm{M} 3$ CETP founders (1 female and 1 male) were obtained. Blood was collected from tail veins of experimental mice (2-6 months old) before and after a high-fat, highcholesterol diet (20\% hydrogenated coconut oil, $0.15 \%$ cholesterol) for 1-2 weeks. CETP activity was determined as described previously (28).

Electrophoresis mobility shift assays. Nuclear extracts were prepared from differentiated 3T3-L1 adipocytes as described previously (29). Double-stranded oligonucleotides used in gel shift assays were synthesized with HindIII overhangs. Nuclear extracts $(\sim 2 \mu \mathrm{g})$ were mixed with $1 \mu \mathrm{L}$ of ${ }^{32} \mathrm{P}$-labeled cpDR4 fragment $(20,000 \mathrm{cpm})$ in a volume of $20 \mu \mathrm{L}$ of binding buffer [75 mM KCl, $20 \mathrm{mM}$ HEPES ( $\mathrm{pH}$ 7.9), $2 \mathrm{mM}$ DTT, $10 \%$ glycerol, $2 \mu \mathrm{g}$ poly(dI-dC), and 30 pmol nonspecific single strand oligonucleotides]. The reactions were incubated at room temperature for 20 minutes, and protein-DNA complexes were resolved on $5 \%$ polyacrylamide gels at $140 \mathrm{~V}$ for 1 hour. For competition experiments, approximately 100 -fold molar excess of unlabeled competitor DNA relative to labeled DNA was added to the reaction mixture before the addition of the labeled probe. In antibody supershift experiments, the gel shift reactions were first incubated for 15 minutes and then 2 $\mu \mathrm{g}$ polyclonal antibodies was added followed by 10 minutes of incubation at room temperature. Polyclonal antibodies against peptides from $\operatorname{LXR} \alpha(\mathrm{P} 20$, sc-1202X), LXR $\alpha / \beta$ (C19, sc-2101X), RXR $\alpha$ (D20, sc$553 \mathrm{X})$, and ROR $\alpha$ (K-20, sc-6063X) were purchased from Santa Cruz Biotechnology, Inc. (Santa Cruz, California, USA).

\section{Results}

A reporter gene controlled by the human CETP proximal promoter is induced by sterol in differentiated 3T3-L1 adipocyte. Luciferase reporter genes controlled by the wild-type CETP promoter from 1 to -570 were transiently transfected into 3T3-L1 preadipocytes or dif-

\section{Figure 3}

(a) Mutational analysis of CETP promoter activity in adipocytes and functionality of the CETP DR4 element in a heterologous TK promoter construct in adipocytes. Mutations were inserted into the CETP promoter with internal deletions $(\Delta 90-370)$ or into the context of the long promoter (M2L-M5L). Mutations M1-M5 are shown in $\mathbf{b}$. Reporter constructs in which reporter genes were controlled by the TK minimal promoter alone (TK-Luc), TK plus 3 copies of the CETP DR4 (3x-cpDR4-TK-Luc), or 3 copies of the 5 ' half site of the DR4 element ( $3 \times 1 / 2$ cpDR4-TK-Luc) were also used to analyze the function of the CETP DR4 element. Fold induction (mean $\pm S D$ ) is shown. The results are from 3-5 independent experiments. ${ }^{*} P<0.0005$ versus mutant constructs; \#P $<0.005$ versus M1-Luc. (b) Sequence of CETP promoter from -380 to -426 . Mutations in this region are shown in italics. The putative DR4 element is shown in bold. M1 is identical to $M 1$ * except that there is an additional single nucleotide mutation in the DR4 element. a

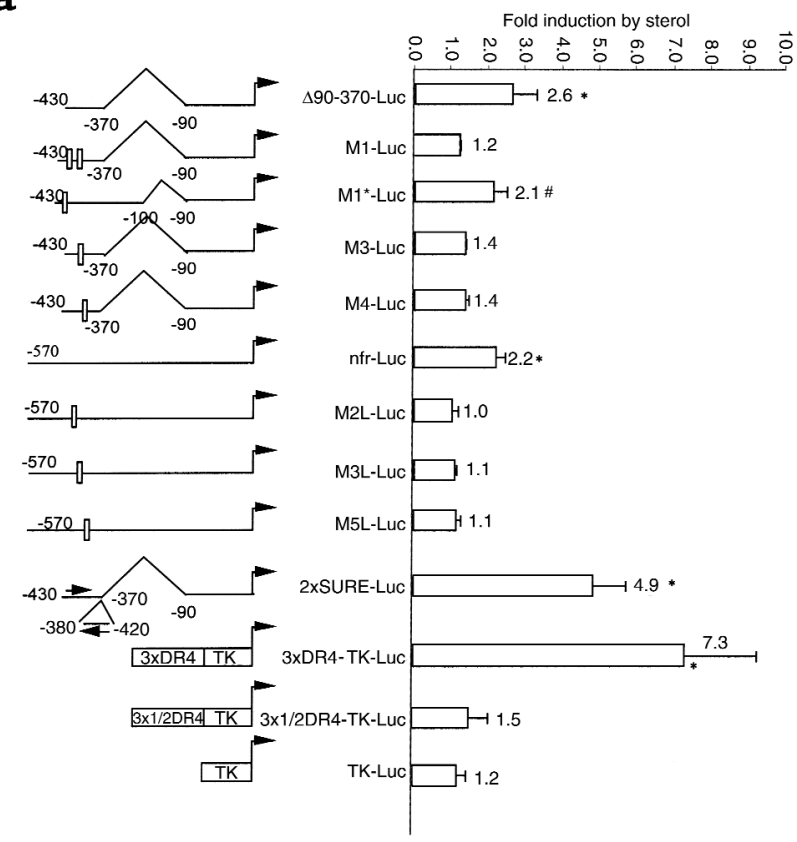

b

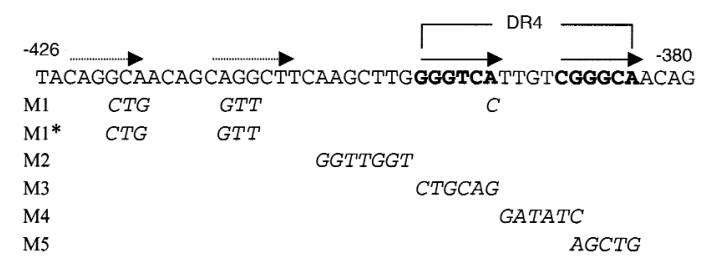


Figure 4

Effect of high-fat, high-cholesterol (HFHC) diet on the plasma CETP activity of mice expressing CETP transgenes controlled by wild-type (NFRCETP), or mutated CETP promoters (MUT3CETP, $M 1^{*}$-CETP, M3-CETP). Five different MUT3-CETP founders, 4 MUT3-CETP F1 mice, 2 $M 1 *$-CETP founders, 2 M3-CETP founders, and 3 control NFR-CETP mice were used. CETP activities (mass) were determined by an isotopic assay using plasma collected before or after an HFHC diet for 7 days. Fold induction (mean \pm SD) of plasma CETP activity by the diet is shown. ${ }^{*} P<0.0005$ versus NFR-CETP.
Fold induction of CETP activity by HFHC diet
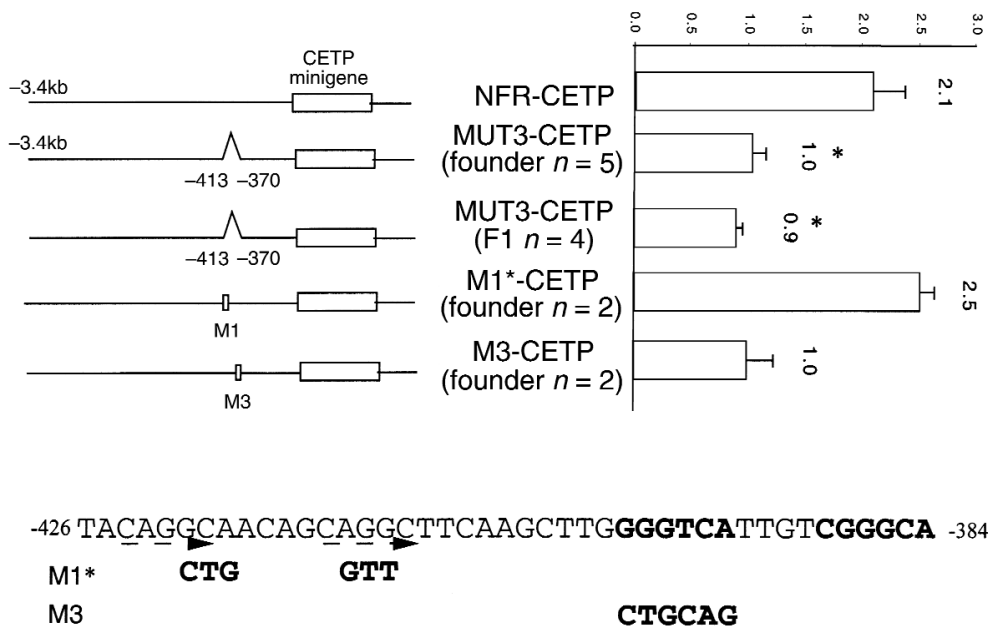

ferentiated adipocytes. The induction of the reporter gene by sterol was analyzed by incubating the cells in the absence or presence of 22(R)-hydroxycholesterol. The luciferase reporter gene activity was induced 2- to 3 -fold by 22(R)-hydroxycholesterol in differentiated adipocytes. However there was no significant sterol induction in preadipocytes (Figure 1a). Similar but less consistent induction was also obtained by 25hydroxycholesterol treatment in adipocytes (data not shown). The CETP promoter contains a sterol regulatory element (SRE), similar to that in the HMG CoA reductase promoter. However, mutation (Mut2) or deletion ( $\triangle \mathrm{SRE}$ ) of the SRE in the CETP promoter did not abolish the sterol induction in adipocytes, consistent with the results in transgenic mice (28). These results suggest that differentiated 3T3-L1 adipocytes can be used as a model to analyze a SURE, distinct from the SRE, in the CETP promoter.

Deletional analysis of the CETP promoter in 3T3-L1 adipocytes. To identify the region containing the SURE, luciferase reporter genes controlled by $5^{\prime}$ truncated CETP promoter elements were transiently transfected into 3T3-L1 adipocytes. Truncation of the region from -570 to -258 abolished the induction by $22(\mathrm{R})$-hydroxycholesterol, suggesting the presence of a SURE in this region (Figure 2). To define further the region containing the SURE, a series of internal deletions in the CETP promoter were generated. Significant sterol upregulation was obtained in the construct containing deletion of the region from -370 to -258 as well as $5^{\prime}$ truncation to -430 (Figure $1 \mathrm{a})$. The -370 to -430 region, linked to the -90 promoter, gave a positive sterol response, whereas deletion of the -370 to -413 sequence abolished sterol induction within the context of the longer -570 bp promoter construct (Figure 2). This result suggests that the region from -370 to -413 is responsible for the positive sterol response in the CETP promoter.

The region from -370 to -430 , which endowed a positive sterol response when linked to the CETP proximal promoter region, contains several sequences that could potentially bind transcription factors (Figure 3b). There is a 6-bp sequence, GGGTCA (-394 to -399), which conforms to the consensus nuclear hormone receptor binding element, PuGG/TTCA. This core sequence and the downstream CGGGCA sequence (although the latter is divergent from the nuclear receptor consensus element) could form a direct repeat separated by 4 nucleotides (DR4). DR4 elements have been shown to bind to nuclear receptors (30). Upstream of the putative DR4 element, there is a direct repeat of a sequence CAGGC separated by 5 nucleotides. To explore whether either of these elements is involved in the positive sterol response, these segments were mutated and the corresponding promoter constructs were evaluated in the luciferase assay (Figure 3a).

Mutations M1-M4 (Figure 3b) were introduced into short promoter reporter constructs (M1-Luc, M3-Luc, and M4-Luc) or were evaluated in the context of the full 570-bp promoter (M2L-Luc, M3L-Luc, and M5L-Luc). The M1 mutation, which disrupted the upstream CAGGC repeats and also has a single nucleotide mutation in the $5^{\prime}$ repeat of the DR4 element, and the M2 mutation, which changed the $5^{\prime}$ flanking sequence of the DR4, abolished the positive sterol response (Figure 3a). However, the $\mathrm{M}^{*}$ mutant, which abolished the upstream repeat without changing the DR4 element, had a normal response (Figure 3a). These results implicate the DR4 element. Other mutations that disrupted the DR4 repeats (M3 and M5) or spacing region (M4) also abolished the positive sterol response. DR4 mutations gave similar results in either the deleted short promoter or the full-length 570-bp promoter. These results suggest that the DR4 element might be the SURE. Consistent with this, a reporter gene controlled by 2 copies of the region containing the SURE (2×SURE-Luc) showed approximately 4 - to 5 -fold induction by sterol, a more robust response than the construct containing 1 copy of the SURE (Figure 3a).

Functionality of the SURE (CETP DR4) in a heterologous $T K$ promoter construct. To confirm further the sterol 
upregulatory function of the DR4 element, DNA fragments containing 3 copies of the CETP DR4 (cpDR4) or the $5^{\prime}$ half site (GGGTCAttgtc) of the DR4 were cloned into a heterologous (TK) promoter construct. Insertion of 3 copies of the CETP DR 4 upstream of TK led to a significant 7-fold sterol induction (Figure 3a, bottom). However, the reporter gene controlled by TK alone or the half element of DR4 was not induced by sterols. This result indicates that the CETP DR4 is sufficient for sterol upregulation and functions as a SURE in a heterologous promoter system.

Evaluation of the SURE in transgenic mice. To determine whether the CETP SURE identified in adipocytes also functions in vivo, we made CETP transgenic mice containing mutations in this element (Figure 4). In control NFR-CETP Tg mice, the high-cholesterol diet caused a significant 2.1-fold induction of plasma CETP activity. Deletion of the -413 to -370 region abolished this response, as shown in 5 different founders and in F1 mice from 1 founder. Transgenic founders bearing mutations in the upstream direct repeat within this region $\left(\mathrm{M} 1^{*}\right.$, Figure $\left.3 \mathrm{~b}\right)$ showed a normal response to the high-cholesterol diet, whereas founders with mutations in the first repeat of the DR4 element (M3, Figure 3b) had no response to the diet. These findings parallel the in vitro results and show that the DR4 element is involved in the response of the human CETP gene in vivo.

Specific protein-DNA interaction involving the CETP DR4 element. DNA mobility shift experiments were carried out to analyze transcription factors binding to the region containing the SURE. A 24-bp DNA fragment (cpDR4), corresponding to the putative DR4 element and its flanking sequence $(-380$ to $-403)$ in the CETP promoter, was incubated with nuclear extracts from 3T3-L1 adipocytes. Excess cold F2 fragments, which consists of only the $5^{\prime}$ half site of DR4, was also included to compete away the binding of proteins to the DR4 half site (data not shown). A major band (P1) (Figure 5a, lane $1)$, representing protein-DNA complexes, was abolished by addition of excess unlabeled cpDR4 (Figure 5a, lane 2). Mutations in the 2 repeats of the DR4 elements (M3, M4, M5, and M1) abolished or reduced the binding of P1, indicating that P1 represents proteins binding to the DR4 (Figure 5a, lanes 4-7). The sequence flanking the DR4 also seems important for the binding of $\mathrm{P} 1$, as $\mathrm{M} 2$, which changed the upstream flanking sequence, competed poorly for the P1

Figure 5 binding (Figure 5a, lane 3). A short fragment, F6, which represents a truncated DR4, containing the $5^{\prime}$ repeat and spacing region, was unable to compete for $\mathrm{P} 1$ binding (Figure 5a, lane 8 ). These results suggest that $\mathrm{P} 1$ protein complex binds to CETP DR4 element and not to the half site of this element. The mutations (M1-M4) that decreased the binding of DNA to P1 protein complex also abolished the sterol upregulation in reporter assays (Figure $3 \mathrm{a}$ ), indicating that the $\mathrm{P} 1$ protein complex probably contains the transcription factors that mediate the sterol upregulation.

Characterization of the transcription factors that bind to the CETP SURE. Two nuclear receptors, LXR $\alpha$ and LXR $\beta$, are known to be activated by certain hydroxysterols and to bind DR4 elements in heterodimeric complexes with RXR $\alpha$ (31). To determine whether the P1 protein complex that binds to the CETP DR4 contains LXR and RXR $\alpha$, we used antibodies against LXR or RXR in gel shift assays (Figure 5b). Polyclonal antibodies against the COOH-terminal 19 amino acids (424-442) of $\mathrm{LXR} \alpha / \beta$ reduced the intensity of the P1 band (Figure $5 \mathrm{~b}$, lane 2) and also gave a weak supershifted band, suggesting the P1 complex contains LXR $\alpha$ and/or $\operatorname{LXR} \beta(22)$. Another antibody (P20), which is against the 20 amino acids (370-389) near the $\mathrm{COOH}$-terminus of LXR $\alpha$, blocked the binding of the proteins to DNA (Figure 5b, lane 3). Two supershifted bands were observed in the presence of RXR $\alpha$ antibody (Figure 5b, lane 4), suggesting that the protein complex also contains RXR $\alpha$. It is not known why RXR $\alpha$ antibody typically produces 2 supershifted bands. A control antibody

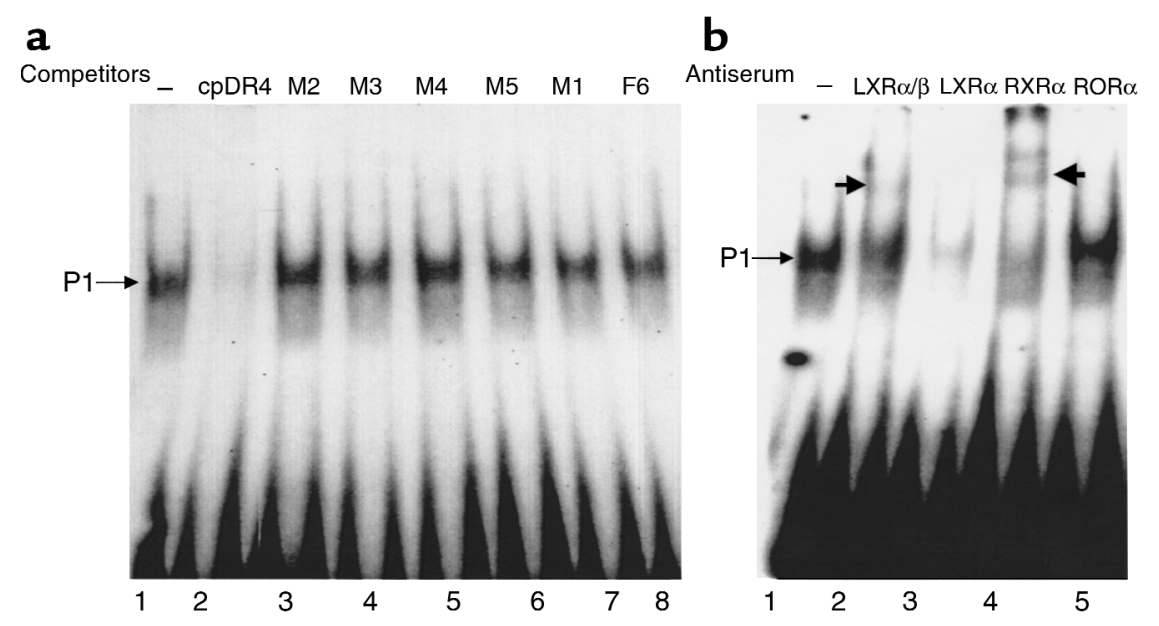

(a) Gel mobility shift analysis to identify the proteins that bind to the DR4 element in the CETP promoter. A 24-bp fragment containing the CETP DR4 (cPDR4) (-380 to -403) was labeled and incubated with nuclear extracts from differentiated 3T3-L1 adipocytes in the presence of excess F2 fragment (CETP DR4 half site) to abolish nonspecific factors binding to the DR4 half site. Various cold competitor fragments were included as indicated, and shifted bands were resolved in a $5 \%$ polyacrylamide gel. F6 consists of only the $5^{\prime}$ half site and spacing region of the DR4 element. M1-M5 mutations are shown in Figure 3b. The arrow indicates the P1 complex. (b) The protein complex binding to the CETP DR4 contains an LXR/RXR heterodimer. Gel shift experiments were carried out in the absence (lane 1) or presence of $2 \mu \mathrm{g}$ polyclonal antibodies against $L X R \alpha / \beta$, $\mathrm{LXR} \alpha$, or RXR $\alpha$ (lanes 2-4), or ROR $\alpha$ (lane 5). The arrow indicates the P1 band. The bands supershifted by RXR $\alpha$ or LXR antibodies are indicated by thick arrows. 


\section{Figure 6}

Additive effects of sterols and 9-cis retinoic acid on CETP promoter activity, mediated via the SURE. Reporter constructs that contain the wild-type CETP promoter ( $\triangle 90-370$-Luc) or promoters with mutations in the DR4 element (M3-Luc) (a) or 3 copies of the CETP DR4 in a heterologous TK promoter (3xcpDR4-TK-Luc) (b) were transfected into differentiated 3T3-L1 adipocytes. Transfected cells were then cultured in LPDS medium in the presence of $4 \mu \mathrm{g} / \mathrm{mL} 22(\mathrm{R})$ hydroxycholesterol, $1 \mu \mathrm{M}$ 9-cis retinoic acid or both. The fold induction (mean \pm SD) from 3 independent experiments is shown except for M2L-luc and M4-Luc, which are in duplicate, and 3xcpDR4-TKLuc, which is in triplicate.

(against ROR $\alpha$ ) had no effect on the P1 complex (Figure 5b, lane 5). Furthermore, The P1 band was competed away by the Cyp7a LXRE, which is known to bind LXR $\alpha$ (19) (data not shown). These data indicate that the P1 complex contains LXR $\alpha$ and RXR $\alpha$. However, these results do not exclude the possibility that LXR $\beta$ is also present in this complex.

LXR $\alpha$ mRNA was found to be markedly induced in differentiated 3T3-L1 adipocyte cells (Figure 1b). The induction of LXR $\alpha$ during adipocyte differentiation is correlated with the appearance of sterol induction of the CETP promoter in differentiated adipocytes (Figure 1a). However, LXR $\beta$ mRNA expression showed little change during differentiation (Figure 1b).

Effect of 9-cis retinoic acid on CETP promoter activity. Gel shift assay data suggest that LXR/RXR $\alpha$ binds to the CETP DR4. It has been shown that RXR $\alpha$ in the LXR/RXR complex plays an active role, in that $\mathrm{RXR} \alpha / \mathrm{LXR}$ heterodimers can be activated by either RXR ligands (i.e., 9-cis retinoic acid), LXR ligands [i.e., 22-(R)hydroxycholesterol], or additively by both LXR and $\mathrm{RXR} \alpha$ ligand $(21,23,31)$. To find out whether RXR $\alpha$ is involved in the function of the CETP SURE, we analyzed the effect of 9-cis retinoic acid on the CETP promoterreporter activity (Figure 6a). The expression of the reporter luciferase controlled by the CETP promoter $(\Delta 90-370-\mathrm{Luc})$ was induced approximately 2 -fold in the presence of 9-cis retinoic acid. An additive induction was observed in the presence of both 22(R)-hydroxycholesterol and 9-cis retinoic acid. Mutation of the DR4 element (M3-M5) and the 5' flanking sequence (M2) abolished both the retinoic acid and sterol induction. Furthermore, the expression of a reporter gene controlled by 3 copies of the DR 4 in a heterologous TK promoter (3xcpDR4-TK-Luc) was highly induced by 9-cis retinoic acid and 22(R)-hydroxycholesterol, and an additive effect ( 23-fold) was also obtained (Figure $6 \mathrm{~b}$ ). These results show that $\mathrm{RXR} \alpha$ is functionally involved in the upregulation of the CETP promoter and that it acts on the DR4 element.

Transactivation of CETP promoter by $L X R \alpha$ and $L X R \beta$. To evaluate further the role of LXRs in sterol activation of the CETP promoter, we analyzed the ability of LXR $\alpha$ and LXR $\beta$ to transactivate the CETP promoter by cotransfecting the CETP promoter-reporter with

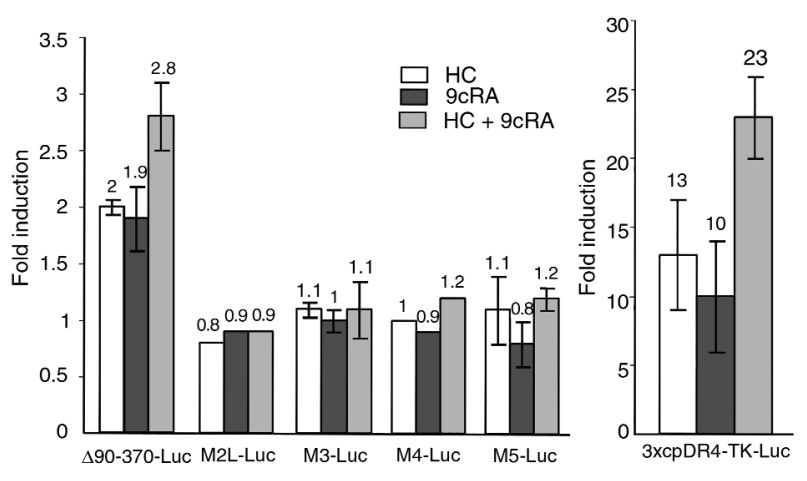

LXR $\alpha / R X R \alpha$ or LXR $\beta / R X R \alpha$ in CV- 1 cells (Figure 7). Transfection of $L X R \alpha / R X R \alpha$ or $L X R \beta / R X R \alpha$ increased the basal expression of the luciferase reporter controlled by the CETP promoter ( $\triangle 90-370$-luc) by approximately 2 - or 5 -fold, respectively. With sterols, the induction significantly increased to approximately 7 - to 10 -fold in cells expressing LXR $\alpha / R X R \alpha$ or LXR $\beta / R X R \alpha$. Mutation of the DR4 element (M3) abolished transactivation by LXRs. LXRs also markedly transactivated the promoter containing 3 copies of the CETP DR4 (SURE). Expression of LXR $\alpha / R X R \alpha$ or $L X R \beta / R X R \alpha$ increased the basal activity of $3 x c p D R 4-T K$ promoter by approximately 3 to 5 -fold and dramatically induced expression (17- to 18 -fold) in the presence of sterols. These results indicate that LXR $\alpha$ or LXR $\beta$ can mediate sterol induction of the CETP gene via the DR4 element.

\section{Discussion}

We found that differentiated 3T3-L1 adipocytes could be used as an in vitro model to study the sterol induction of the human CETP promoter. This enabled us to identify in the human CETP promoter a novel SURE that consists of a variant DR4 (2 direct repeats separated by 4 nucleotides) nuclear hormone receptor binding sequence. Importantly, the DR4 element not only functions as a SURE in vitro but also mediates the induction of human CETP transgene expression by dietary cholesterol in transgenic mice. The DR4 element binds LXR $\alpha$ and LXR $\beta$ and is activated in a sterol-sensitive fashion. These findings broaden the biologic role of LXRs beyond the regulation of bile acid synthesis and suggest that LXR $\alpha$ and possibly LXR $\beta$ also play a role in the regulation in HDL metabolism. LXRs may help to coordinate HDL cholesterol ester catabolism with hepatic excretion of cholesterol by conversion to bile salts.

The 5' half site (GGGTCA) of the CETP SURE (DR4 element) conforms to the consensus nuclear hormone receptor half site, A/GGG/TTCA (30). Mutation of the site to 'GGGTCC' abolished protein binding and sterol induction (Figures 3 and 5). A similar mutation also abolished the binding of LXR $\alpha / R X R \alpha$ to the LXRE (21). However the $3^{\prime}$ half site, $\underline{C G G} \underline{G C A}$, is divergent, as 2 bases (underlined) are different from the consensus sequence. In contrast, both Cyp7a LXRE DR4 repeats, T/AGG/TTCA, con- 
form to the nuclear hormone receptor consensus sequence (19). These results imply that LXRs might be able to bind DR4 elements that are divergent from the consensus sequence, such as the CETP DR4 SURE. The activation of the CETP DR 4 element by LXR $\beta$ might be related to its divergent sequence (Figure 7).

Although LXR $\alpha$ and LXR $\beta$ are about $77 \%$ identical in DNA binding and ligand binding domain, they are likely to have differential effects in vivo. Whereas, LXR $\alpha$ is predominantly expressed in central organs such as liver and adipose (21), LXR $\beta$ is ubiquitously expressed (22). The Cyp7a promoter was shown to be transactivated efficiently by LXR $\alpha$ but not by LXR $\beta$ (19). Furthermore, disruption of LXR $\alpha$ in mice specifically abolished the dietary cholesterol induced Cyp7a expression, whereas the basal expression was not changed (24). LXR $\beta$ expression in $\operatorname{LXR} \alpha^{-/-}$mice was unchanged, indicating that $\operatorname{LXR} \beta$ did not compensate for the function of LXR $\alpha$ (24). No targets of LXR $\beta$ have yet been found, and the LXR $\beta$ knockout mice showed no obvious abnormality (31).

Here we show that CETP is well transactivated by LXR $\alpha$ and LXR $\beta$, suggesting they might both have a role in regulating CETP expression in vivo (Figure 7). LXR $\alpha$ expression is low in preadipocytes and is highly induced in 3T3-L1 adipocytes (Figure 1b). Interestingly, the differentiation cocktail contains IBMX, which increases the levels of cAMP and which has recently been suggested to mediate renin gene induction via $\operatorname{LXR} \alpha$ (32). The dependence of sterol induction of the CETP promoter on adipocyte differentiation (Figure 1a) might be due to the induction of LXR $\alpha$ expression during differentiation. A role for LXR $\alpha$ is also suggested by the fact that the major tissues expressing the human CETP gene are identical to those with highest LXR $\alpha$ expression (liver, spleen, kidney, adipose, small intestine) $(13,21)$. These data strongly suggest the involvement of LXR $\alpha$ in the sterol regulation of CETP. The expression of the reporter gene controlled by the CETP promoter or CETP SURE was transactivated by LXR $\beta$ at least as efficiently as by LXR $\alpha$ (Figure 7). However, LXR $\beta$ is highly expressed in preadipocytes in which no significant sterol induction was obtained. It remains possible that activation of LXR $\beta$ may need differentiation-related post-transcriptional modifications (e.g., phosphorylation) or cofactors. The existence of an alternative regulatory mechanism for the CETP gene, possibly involving LXR $\beta$, is also suggested by the fact that CETP mRNA levels were increased in hypercholesterolemic apoE null mice (apoE0), whereas Cyp7a mRNA levels remained unchanged (ref. 5 and our unpublished results). Although LXR $\alpha$ and LXR $\beta$ can transactivate the CETP promoter in cultured cells, we cannot exclude the possibility that other unknown LXR homologues activated by sterols may mediate the sterol induction of the CETP DR4 element in vivo.

The induction of the CETP gene in vivo by dietary cholesterol and endogenous hypercholesterolemia may be due to an increased hepatic content of 24(S),25epoxycholesterol or other hydroxycholesterols [such as 24(S)-hydroxycholesterol] that are ligands for LXRs.
Dietary cholesterol is known to increase the hepatic content of 24(S),25- epoxycholesterol, which is synthesized from squalene by a shunt pathway (33). The shunt pathway may be more active due to suppression of conversion of squalene to cholesterol on the highcholesterol diet. 24(S)-hydroxycholesterol is a direct metabolite of cholesterol (34).

Several genes involved in distinct steps of reverse cholesterol transport (RCT) are upregulated by sterols, including $A P O E, A B C 1$, CETP, and CyP7a. APOE and $A B C 1$ are induced in cholesterol-loaded macrophages $(15,16)$ and may promote RCT by enhancing cholesterol efflux from macrophage to apoA-I or HDL. In turn, CETP facilitates clearance of HDL CE by the liver and then Cyp7a increases the catabolism of cholesterol in the liver (35). It has been suggested that these genes might be coordinately regulated (25). The data from the present study and that of Peet et al. (24) show that both Cyp7a and CETP are upregulated by LXR $\alpha$ in response to a high-cholesterol diet. The disruption of LXR $\alpha$ in mice fed a chow diet also led to increases in the mRNA levels of several genes involved in cholesterol biosynthesis. These included HMG CoA reductase, HMG CoA synthase, and SREBP-2, a transcription factor that regulates the expression of cholesterol biosynthetic enzymes (36). We postulate that LXRs may also play a critical role in regulating the expression of several genes involved in RCT. Suppression of hepatic cholesterol biosynthesis while increasing RCT may represent 2 facets of the response to tissue cholesterol overload, coordinated in part by LXRs.

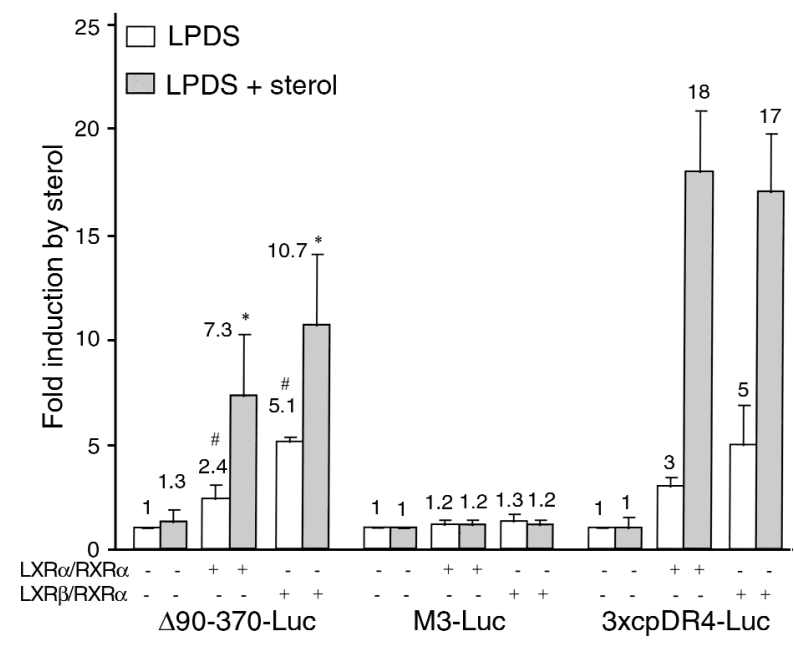

Figure 7

Transactivation of the CETP promoter or a synthetic promoter containing 3 copies of the DR4 from the CETP promoter (3xcpDR4) by $L X R \alpha$ and LXR $\beta$ in CV- 1 cells. CV- 1 cells were transfected with $\Delta 90-370$ Luc, M3-luc, or 3XcpDR4-TK-Luc with or without cotransfection with $L X R \alpha / R X R \alpha$ or $L X R \beta / R X R \alpha$. The transfected cells were then cultured in LPDS $\pm 4 \mu \mathrm{g} / \mathrm{mL} 22$ (R)-hydroxycholesterol. Three experiments were carried out in duplicate for $\Delta 90-370$-Luc and 3 XcpDR4-TK-Luc. Two experiments were carried out for M3-Luc. $\# P<0.001$ versus the same condition without cotransfection with $\mathrm{LXR} \alpha / \mathrm{RXR} \alpha$ or $\mathrm{LXR} \beta / \mathrm{RXR} \alpha$. ${ }^{*} P<0.05$ versus the same conditions in the absence of sterols. 


\section{Acknowledgments}

This work was supported by a grant from the National Institutes of Health (HL-54591). We thank A. Walsh and members in the animal facility at Rockefeller University for generation of transgenic mice. We are grateful to D. Mangelsdorf for sending us the CMX-hRXRa, CMX-hLXRa, and TK-Luc plasmids. We thank C. Glass for critical comments.

1. Tall, A. 1995. Plasma lipid transfer proteins. Annu. Rev. Biochem. 64:235-257.

2. Brown, M.L., et al. 1989. Molecular basis of lipid transfer protein deficiency in a family with increased high-density lipoproteins. Nature. 342:448-451.

3. Inazu, A., et al. 1994. Genetic cholesteryl ester transfer protein deficiency caused by two prevalent mutations as a major determinant of increased levels of high density lipoprotein cholesterol. J. Clin. Invest. 94:1872-1882

4. Agellon, L.B., et al. 1991. Reduced high density lipoprotein cholesterol in human cholesteryl ester transfer protein transgenic mice. $J$. Biol. Chem. 266:10796-10801.

5. Masucci-Magoulas, L., et al. 1996. Profound induction of hepatic cholesteryl ester transfer protein transgene expression in apolipoprotein $\mathrm{E}$ and low density lipoprotein receptor gene knockout mice. A novel mechanism signals changes in plasma cholesterol levels. J. Clin. Invest. 97:154-161.

6. Zhong, S., et al. 1996. Increased coronary heart disease in JapaneseAmerican men with mutation in the cholesteryl ester transfer protein gene despite increased HDL levels. J. Clin. Invest. 97:2917-2923.

7. Hayek, T., et al. 1995. Decreased early atherosclerotic lesions in hypertriglyceridemic mice expressing cholesteryl ester transfer protein transgene. J. Clin. Invest. 96:2071-2074.

8. Collet, X., et al. 1999. Remodeling of HDL by CETP in vivo and by CETP and hepatic lipase in vitro results in enhanced uptake of HDL CE by cells expressing scavenger receptor B-I. J. Lipid Res. 40:1185-1193.

9. Francone, O.L., Royer, L., and Haghpassand, M. 1996. Increased prebeta-HDL levels, cholesterol efflux, and LCAT-mediated esterification in mice expressing the human cholesteryl ester transfer protein (CETP) and human apolipoprotein A-I (apoA-I) transgenes. J. Lipid Res. 37:1268-1277.

10. Quinet, E.M., et al. 1990. Atherogenic diet increases cholesteryl ester transfer protein messenger RNA levels in rabbit liver. J. Clin. Invest. 85:357-363.

11. Jiang, X.C., et al. 1991. Mammalian adipose tissue and muscle are major sources of lipid transfer protein mRNA. J. Biol. Chem. 266:4631-4639.

12. Gauthier, B., Robb, M., and McPherson, R. 1999. Cholesteryl ester transfer protein gene expression during differentiation of human preadipocytes to adipocytes in primary culture. Atherosclerosis. 142:301-307.

13. Jiang, X.C., Agellon, L.B., Walsh, A., Breslow, J.L., and Tall, A. 1992. Dietary cholesterol increases transcription of the human cholesteryl ester transfer protein gene in transgenic mice. Dependence on natural flanking sequences. J. Clin. Invest. 90:1290-1295.

14. Martin, L.J., et al. 1993. Cholesteryl ester transfer protein and high density lipoprotein responses to cholesterol feeding in men: relationship to apolipoprotein E genotype. J. Lipid Res. 34:437-446.

15. Mazzone, T., Basheeruddin, K., and Poulos, C. 1989. Regulation of macrophage apolipoprotein E gene expression by cholesterol. J. Lipid. Res. 30:1055-1064.

16. Langmann, T., et al. 1999. Molecular cloning of the human ATPbinding cassette transporter 1 (hABC1): evidence for sterol-dependent regulation in macrophages. Biochem. Biophys. Res. Commun.
257:29-33

17. Janowski, B.A., Willy, P.J., Devi, T.R., Falck, J.R., and Mangelsdorf, D.J. 1996. An oxysterol signalling pathway mediated by the nuclear receptor LXR $\alpha$. Nature. 383:728-731.

18. Janowski, B.A., et al. 1999. Structural requirements of ligands for the oxysterol liver X receptors LXR $\alpha$ and LXR $\beta$. Proc. Natl. Acad. Sci. USA. 96:266-271.

19. Lehmann, J.M., et al. 1997. Activation of the nuclear receptor LXR by oxysterols defines a new hormone response pathway. J. Biol. Chem. 272:3137-3140.

20. Lala, D.S., et al. 1997. Activation of the orphan nuclear receptor steroidogenic factor 1 by oxysterols. Proc. Natl. Acad. Sci. USA. 94:4895-4900.

21. Willy, P.J., et al. 1995. LXR, a nuclear receptor that defines a distinct retinoid response pathway. Genes Dev. 9:1033-1045.

22. Song, C., Kokontis, J.M., Hiipakka, R.A., and Liao, S. 1994. Ubiquitous receptor: a receptor that modulates gene activation by retinoic acid and thyroid hormone receptors. Proc. Natl. Acad. Sci. USA. 91:10809-10813

23. Teboul, M., et al. 1995. OR-1, a member of the nuclear receptor superfamily that interacts with the 9-cis-retinoic acid receptor. Proc. Natl. Acad. Sci. USA. 92:2096-2100.

24. Peet, D.J., et al. 1998. Cholesterol and bile acid metabolism are impaired in mice lacking the nuclear oxysterol receptor LXR alpha. Cell. 93:693-704

25. Oliveira, H.C.F., et al. 1996. Human cholesteryl ester transfer protein gene proximal promoter contains dietary cholesterol positive responsive elements and mediates expression in small intestine and periphery while predominant liver and spleen expression is controlled by 5 distal sequences. Cis-acting sequences mapped in transgenic mice. $J$. Biol. Chem. 271:31831-31838.

26. Agellon, L.B., Zhang, P., Jiang, X.C., Mendelsohn, L., and Tall, A.R. 1992. The CCAAT/enhancer-binding protein trans-activates the human cholesteryl ester transfer protein gene promoter. J. Biol. Chem. 267:22336-22339.

27. Kim, J.B., and Spiegelman, B.M. 1996. ADD1/SREBP1 promotes adipocyte differentiation and gene expression linked to fatty acid metabolism. Genes Dev. 10:1096-1107.

28. Chouinard, R.A., Jr., Luo, Y., Osborne, T.F., Walsh, A., and Tall, A.R 1998. Sterol regulatory element binding protein-1 activates the cholesteryl ester transfer protein gene in vivo but is not required for sterol up-regulation of gene expression. J. Biol. Chem. 273:22409-22414

29. Dignam, J.D., Martin, P.L., Shastry, B.S., and Roeder, R.G. 1983 Eukaryotic gene transcription with purified components. Methods Enzymol. 101:582-598

30. Mangelsdorf, D.J., et al. 1995. The nuclear receptor superfamily: the second decade. Cell. 83:835-839.

31. Peet, D.J., Janowski, B.A., and Mangelsdorf, D.J. 1998. The LXRs: a new class of oxysterol receptors. Curr. Opin. Genet. Dev. 8:571-575.

32. Tamura, K., and Chen, Y.E. 1999. LXRa is a novel cAMP responsive transcriptional factor in renin gene expression. Circulation. 100:1867. (Abstr.)

33. Nelson, J.A., Steckbeck, S.R., and Spencer, T.A. 1981. Biosynthesis of 24,25-epoxycholesterol from squalene 2,3;22,23-dioxide. J. Biol. Chem. 256:1067-1068.

34. Kliewer, S.A., Lehmann, J.M., and Willson, T.M. 1999. Orphan nuclear receptors: shifting endocrinology into reverse. Science. 284:757-760.

35. Russell, D.W., and Setchell, K.D. 1992. Bile acid biosynthesis. Biochemistry. 31:4737-4749.

36. Brown, M.S., and Goldstein, J.L. 1997. The SREBP pathway: regulation of cholesterol metabolism by proteolysis of a membrane-bound transcription factor. Cell. 89:331-340.

37. Seol, W., Choi, H.S., and Moore, D.D. 1995. Isolation of proteins that interact specifically with the retinoid $\mathrm{X}$ receptor: two novel orphan receptors. Mol. Endocrinol. 9:72-85 